\title{
An Accurate and Low Complex Channel Estimator for OFDM WiMAX
}

\author{
Christian Mehlführer, Sebastian Caban, and Markus Rupp \\ Institute of Communications and Radio-Frequency Engineering \\ Vienna University of Technology \\ Gusshausstrasse 25/389, A-1040 Vienna, Austria \\ Email: \{chmehl, scaban, mrupp $\} @$ nt.tuwien.ac.at \\ Web: http://www.nt.tuwien.ac.at/rapid-prototyping
}

\begin{abstract}
In this paper, we present an approximate LMMSE (ALMMSE) channel estimator for OFDM WiMAX. The ALMMSE estimator utilizes the inherent correlation between the channels of neighboring subcarriers to improve the least squares estimate by a spectral smoothing filter. We show that the covariance matrix of the channel, required for the calculation of the spectral smoothing filter, can be estimated from the LS channel estimate of the same receive frame. The complexity of the proposed ALMMSE estimator is considerably lower than the complexity of the exact LMMSE estimator while the performance loss is only about $0.3 \mathrm{~dB}$ in SNR. Our evaluation of the channel estimator performance is based on outdoor measurements in realistic WiMAX scenarios.
\end{abstract}

\section{INTRODUCTION}

Modern wireless communication systems-like the IEEE 802.16-2004 WiMAX [1] system considered in this worktypically rely on training symbol based channel estimation. An accurate channel estimate is of utmost importance for equalization at the receiver and thus directly affects the achieved data throughput. The accuracy of the channel estimate is linked to the number of transmitted training symbols. However, a large number of training symbols also lowers the achievable data throughput since the training symbol energy cannot be used for transmitting data symbols. A very limited number of training symbols have thus to be used as efficiently as possible. Very good channel estimator performance can be obtained by linear minimum mean squared error (LMMSE) estimation which takes the channel covariance into account. Unfortunately, the direct implementation of the LMMSE estimator is of prohibitive complexity for real-time implementations. If the noise variance and the channel covariance are unknown (and no correlation between the channels of neighboring subcarriers has to be assumed), the LMMSE estimator degrades to a least squares (LS) estimator, yielding only poor performance at low complexity. Alternatively, low complexity LMMSE channel estimation using FFT and IFFT operations was previously described in e.g. [2]. However, this estimator relies on perfect a-priori knowledge of the noise variance and the channel covariance. In this work, we propose an approximate LMMSE (ALMMSE) estimator that post-processes the LS channel estimate using a spectral smoothing filter. The filter coefficients depend on the noise variance and the channel covariance which can be directly obtained from the LS channel estimate in the same receive frame. For the special case of IEEE 802.16-2004 WiMAX, the ALMMSE estimator requires only the inversion of one $5 \times 5$ matrix compared to the inversion of a $100 \times 100$ matrix in the direct implementation of the LMMSE estimator. Although our derivation of the ALMMSE estimator in this work is based on the actual WiMAX training sequences, it can be adapted to other OFDM based transmission standards.

The paper is organized as follows. Section II describes the training symbol structure of the WiMAX standard and the resulting system model. The LS, LMMSE, and ALMMSE channel estimators are derived in Section III. Additionally, we define a "perfect" channel estimator that we use as a benchmark. In Section IV, we assess the implementation complexity of the channel estimators. Our measurement setup for the performance evaluation of the channel estimators is described in Section V. The results are presented in Section VI. Finally, we draw some conclusions in Section VII.

\section{SySTEM MODEL}

In this section, the structure of the training sequence in the IEEE 802.16-2004 WiMAX standard [1] and the resulting system model are described.

Since the 2004 WiMAX standard is dedicated to static and quasi-static scenarios, it only defines one OFDM training symbol in the preamble of every frame. At the first transmit antenna, the energy of the training is only distributed on the even subcarriers while zeros are transmitted at the odd subcarriers. At the second transmit antenna, only the odd subcarriers are utilized for the training symbols. This structure allows to estimate the links of the MIMO channel individually by using independent SISO channel estimators if the spatial correlation is neglected. We will assume in the following that the spatial correlation is small in order to derive a very low complexity channel estimator that can be applied to every SISO link of the MIMO system individually.

The received OFDM training symbol $\mathbf{r}$ at one receive antenna can be written as

$$
\mathbf{r}=\mathbf{T h}+\mathbf{v},
$$


where the diagonal matrix $\mathbf{T}$ comprises the $N=100$ training symbols $^{1}$ of one transmit antenna on the main diagonal, the vector $\mathbf{h}$ contains the channel coefficients of the SISO link between the transmit antenna and the receive antenna to be estimated, and $\mathbf{v}$ is additive white Gaussian noise at the receive antenna. Note that due to the given training sequence a direct channel coefficient estimation is only possible for the even subcarriers of transmit antenna one. The channel coefficients of the odd subcarriers have to be obtained by interpolation. The corresponding holds true for the even subcarriers of the second transmit antenna.

\section{Channel Estimators}

In this section, the different types of channel estimators considered in this paper are explained.

\section{A. LS Estimator}

The least squares channel estimator for the system model given by Equation (1) can be easily shown to be given by

$$
\hat{\mathbf{h}}^{\mathrm{LS}}=\left(\mathbf{T}^{H} \mathbf{T}\right)^{-1} \mathbf{T}^{H} \mathbf{r} .
$$

As defined in the standard, the training symbols on all subcarriers have energy two. Due to the diagonal structure of $\mathbf{T}$ the estimator is simplified to

$$
\hat{\mathbf{h}}^{\mathrm{LS}}=\frac{1}{4} \mathbf{T}^{H} \mathbf{r} .
$$

The LS estimator is therefore of very low complexity (only one complex multiplication per channel coefficient) but unfortunately yields poor performance as shown in the measurement results.

\section{B. "Perfect" Channel Estimator}

Since our evaluation is based on over-the-air transmissions, the true channel is unknown but can be estimated with very high accuracy by using all transmitted data symbols of one frame (47 OFDM data symbols in contrast to one OFDM training symbol) in a simple LS estimator. Of course, in a real system the receiver does not have any knowledge about the transmitted data symbols. This genie driven estimatorwe call it the "perfect" channel estimator in the following-is used as a benchmark for all other estimators in this paper.

\section{LMMSE Estimator}

The LMMSE estimator is derived by minimizing the quadratic estimation error:

$$
\hat{\mathbf{h}}^{\text {LMMSE }}=\arg \min _{\hat{\mathbf{h}}} \mathrm{E}\left\{\|\hat{\mathbf{h}}-\mathbf{h}\|_{2}^{2}\right\} .
$$

Using the linearity constraint for the estimator we obtain the general solution [3]

$$
\hat{\mathbf{h}}^{\text {LMMSE }}=\mathbf{R}_{\mathbf{h r}} \mathbf{R}_{\mathbf{r r}}^{-1} \mathbf{r},
$$

${ }^{1}$ The IEEE 802.16-2004 WiMAX standard specifies the OFDM physical layer to comprise 256 subcarriers. These 256 subcarriers consist of 200 data+pilot carriers, 55 guard band carriers, and one zero DC carrier. Since the training symbols only allocate every second of the 200 data+pilot carriers, we end up with $N=100$. with the cross-correlation matrix $\mathbf{R}_{\mathbf{h r}}$ and the auto-correlation matrix $\mathbf{R}_{\mathbf{r r}}$. Assuming that the additive noise is uncorrelated with variance $\sigma_{v}^{2}$, the LMMSE estimator according to the system model is given by

$$
\begin{aligned}
\hat{\mathbf{h}}^{\text {LMMSE }} & =\mathbf{R}_{\mathbf{h h}} \mathbf{T}^{H}\left(\mathbf{T R}_{\mathbf{h h}} \mathbf{T}^{H}+\mathbf{R}_{\mathbf{v v}}\right)^{-1} \mathbf{r}= \\
& =\mathbf{R}_{\mathbf{h h}}\left(\mathbf{R}_{\mathbf{h h}}+\frac{\sigma_{v}^{2}}{4} \mathbf{I}\right)^{-1} \frac{1}{4} \mathbf{T}^{H} \mathbf{r}= \\
& =\mathbf{R}_{\mathbf{h h}}\left(\mathbf{R}_{\mathbf{h h}}+\frac{\sigma_{v}^{2}}{4} \mathbf{I}\right)^{-1} \hat{\mathbf{h}}^{\mathrm{LS}}=\mathbf{F} \hat{\mathbf{h}}^{\mathrm{LS}} .
\end{aligned}
$$

The LMMSE estimate is therefore obtained by post-processing (filtering) the LS channel estimate with the matrix F. Since the direct implementation of Equation (6) requires the inversion of a large (WiMAX: $100 \times 100)$ matrix, it is of very high computational complexity. Another problem of the LMMSE estimator is the computation of the full correlation matrix $\mathbf{R}_{\mathbf{h h}}$. Usually this matrix is calculated by averaging over $N_{c}$, previously estimated channels $\hat{\mathbf{h}}^{(i)}$ :

$$
\mathbf{R}_{\mathbf{h h}}=\mathrm{E}\left\{\mathbf{h h}^{H}\right\} \approx \hat{\mathbf{R}}_{\mathbf{h h}}=\frac{1}{N_{\mathrm{c}}} \sum_{i=1}^{N_{\mathrm{c}}} \hat{\mathbf{h}}^{(i)} \hat{\mathbf{h}}^{(i) H} .
$$

For the correct function of the LMMSE channel estimator, $\hat{\mathbf{R}}_{\mathbf{h h}}$ has to be of full rank, requiring that the number of previously observed channel realizations has to be larger than the length of the channel vector $\mathbf{h}\left(N_{\mathrm{c}} \geq N\right)$. The computation of the full correlation matrix therefore requires a large number of previously observed channels which are often not available.

To overcome this problem, an approximate LMMSE estimator with greatly reduced complexity will be introduced in the next section.

\section{Approximate LMMSE Estimator}

One possibility to reduce the complexity of the LMMSE estimator in Equation (6) is to reduce the size of the correlation matrix $\mathbf{R}_{\mathbf{h h}}$ and thus also the size of the filtering matrix $\mathbf{F}$. This can be achieved by partitioning the channel vector $\mathbf{h}$ into $M$ subvectors of length $L$ (with $L \ll N$ and $M=\left\lfloor\frac{N}{L}\right\rfloor$ ): ${ }^{2}$

$$
\mathbf{h}=\left[\mathbf{h}_{1}^{T}, \ldots, \mathbf{h}_{M}^{T}\right]^{T} .
$$

Here, the $m$-th subvector is given by $\mathbf{h}_{m}=$ $\left[h_{L(m-1)+1}, \ldots, h_{L(m-1)+L}\right]^{T}$. The length $L$ can be chosen depending on the coherence bandwidth of the channel or can be set to a fixed value. In the measurement results we will show that a small value of $L=5$ almost achieves the LMMSE performance even in a line-of-sight (LOS) scenario, where the frequency correlation is large.

\section{Approach of [3]}

As pointed out in [3], the channel coefficient subvectors $\mathbf{h}_{m}$ can be estimated by the following low complexity estimator

$$
\hat{\mathbf{h}}_{m}=\mathbf{R}_{\mathbf{h}_{m} \mathbf{h}_{m}}\left(\mathbf{R}_{\mathbf{h}_{m} \mathbf{h}_{m}}+\frac{\sigma_{v}^{2}}{4} \mathbf{I}\right)^{-1} \hat{\mathbf{h}}_{m}^{L S}=\mathbf{F}_{m}^{(L)} \hat{\mathbf{h}}_{m}^{L S} .
$$

${ }^{2}$ We use the symbols $\lceil$.$\rceil and \lfloor$.$\rfloor to represent the ceiling and floor operators,$ respectively. 
However, this approximation suffers from an increased MSE at the edges of the subbands created by the partitioning of the channel vector. In [3] this problem is solved by an overlap technique where the channel vector in Equation (8) is partitioned into overlapping subbands.

The estimator in Equation (9) requires the calculation of a separate filter matrix $\mathbf{F}_{m}^{(L)}$ for every subband $m$ and thus the calculation of many matrix inverses.

\section{Novel approach}

We propose the following approach for approximating the LMMSE channel estimator.

1) Calculation of only one correlation matrix $\hat{\mathbf{R}}_{\mathbf{h h}}^{(L)}$ of dimension $L \times L$. This matrix is obtained by averaging over the correlation matrices $\hat{\mathbf{R}}_{\mathbf{h}_{m} \mathbf{h}_{m}}$.

2) Since the correlation matrix $\hat{\mathbf{R}}_{\mathbf{h h}}^{(L)}$ is independent of the subband index $m$, we only need to calculate one filter matrix $\mathbf{F}^{(L)}$.

3) Application of this single filter matrix $\mathbf{F}^{(L)}$ to improve the LS channel estimate to near LMMSE accuracy.

The single correlation matrix $\hat{\mathbf{R}}_{\mathbf{h h}}^{(L)}$ of size $L \times L$ is therefore obtained as

$$
\hat{\mathbf{R}}_{\mathbf{h h}}^{(L)}=\mathrm{E}\left\{\mathbf{h}_{m} \mathbf{h}_{m}^{H}\right\} \triangleq \frac{1}{M N_{\mathrm{c}}} \sum_{i=1}^{N_{\mathrm{c}}} \sum_{m=1}^{M} \mathbf{h}_{m}^{(i)} \mathbf{h}_{m}^{(i) H} .
$$

Here, the full-rank-condition for $\hat{\mathbf{R}}_{\mathbf{h h}}^{(L)}$ is reduced to $M N_{\mathrm{c}} \geq$ $L$. If, for example, $L=10$ is chosen, $M$ can be calculated as $M=\left\lfloor\frac{N}{L}\right\rfloor=\left\lfloor\frac{100}{10}\right\rfloor=10$, meaning that the number of required channel realizations is only one. In other words, after performing the LS channel estimation we can already find a full rank estimate for the reduced size channel correlation matrix.

The reduced size correlation matrix can now be used to calculate the $L \times L$ filter matrix $\mathbf{F}^{(L)}$ for post processing the LS channel estimate:

$$
\mathbf{F}^{(L)}=\hat{\mathbf{R}}_{\mathbf{h h}}^{(L)}\left(\hat{\mathbf{R}}_{\mathbf{h h}}^{(L)}+\frac{\sigma_{v}^{2}}{4} \mathbf{I}\right)^{-1} .
$$

The approximate LMMSE (ALMMSE) estimator is now given by filtering the LS channel estimate according to

$$
\begin{aligned}
& \hat{h}_{n}^{\text {ALMMSE }}= \\
&= \begin{cases}\mathbf{F}_{n,:}^{(L)} \cdot\left[\hat{h}_{1}^{\mathrm{LS}}, \ldots, \hat{h}_{L}^{\mathrm{LS}}\right]^{T} & ; n \leq \frac{L+1}{2} \\
\mathbf{F}_{\left\lceil\frac{L+1}{2}\right\rceil,:}^{(L)} \cdot\left[\hat{h}_{n-\left\lfloor\frac{L-1}{2}\right\rfloor}^{\mathrm{LS}}, \ldots, \hat{h}_{n-\left\lceil\frac{L-1}{\mathrm{LS}}\right\rceil}^{\mathrm{LS}}\right]^{T} & ; \text { otherwise } \\
\mathbf{F}_{L+n-N,:}^{(L)} \cdot\left[\hat{h}_{N-L+1}^{\mathrm{LS}}, \ldots, \hat{h}_{N}^{\mathrm{LS}}\right]^{T} & ; n \geq N-\frac{L-1}{2}\end{cases}
\end{aligned}
$$

Here, $\mathbf{F}_{n, \text { : }}^{(L)}$ means the $n$-th row of the filter matrix $\mathbf{F}^{(L)}$. The operation performed in Equation (12) is a weighted averaging filter with $L$ coefficients. To avoid transient responses (leading to increased MSE) the LS channel coefficients at the band edges ( $n \leq \frac{L+1}{2}$ and $n \geq N-\frac{L-1}{2}$ ) are filtered with different coefficients (the first and last rows of the matrix $\mathbf{F}^{(L)}$ ).

\section{Complexity Assessment}

The implementation complexity of the LMMSE estimator, our proposed ALMMSE estimator, and the estimator introduced in [3] is mainly determined by the calculation of the matrix inverse which has a complexity of $\mathcal{O}\left(K^{3}\right)$ (for a matrix of dimension $K \times K)$. For full LMMSE estimation the complexity is therefore $\mathcal{O}\left(N^{3}\right)(N=100)$, and for the low complexity estimators (our proposed LMMSE and the estimator in [3]) $\mathcal{O}\left(L^{3}\right)$ (with $\left.L \ll N\right)$. Our proposed estimator, however, requires only the calculation of one such matrix inverse, whereas the estimator of [3]) requires the calculation of $2 M-1$ matrix inverses $\left(M=\left\lfloor\frac{N}{L}\right\rfloor\right)$. For the particular values of $N=100, L=5$, and $M=20$ that show almost the performance of the full LMMSE estimator in our measurements, this corresponds to a complexity decrease by a factor of 39 !

\section{Measurement Setup}

In this section we briefly describe the measurement setup used to obtain the results presented in the next section. A full description of the measurement setup can be found in [4]. For our measurements we used the Vienna MIMO Testbed presented in [5, 6] and a Matlab implementation of the IEEE 802.16-2004 WiMAX standard [7]. The basic features of the testbed are as follows.

- Baseband processing is carried out off-line in MATLAB with floating-point precision. The transmitted and received, down-sampled signals are stored on hard-disk drives (approx. 600 Gbytes per scenario measured).

- Receiver and Transmitter are synchronized by means of rubidium frequency standards and a LAN connection.

- The carrier frequency is $2.5 \mathrm{GHz}$, the bandwidth $5 \mathrm{MHz}$.

- The two transmit antennas are mounted on a pole 16 meters above rooftop [8] in an urban scenario (historic city center of Vienna). When WiMAX is used as a lastmile internet access technology, this transmit antenna position is very realistic for a base station.

- The receive antennas are placed on a step-motor controlled linear XY positioning table that was used to measure at 506 different receive antenna positions. The positioning table was placed in an NLOS and a LOS scenario.

- In the NLOS scenario the table was placed in a nonline of sight connection in the courtyard next to the transmit antenna.

- The LOS scenario was established by placing the table with the receive antennas in the building adjacent to the building where the transmit antennas are mounted.

These scenarios represent different locations of a user accessing the internet via the WiMAX connection and are thus very practical.

- All seven adaptive modulation and coding (AMC) schemes of WiMAX were transmitted successively. The 


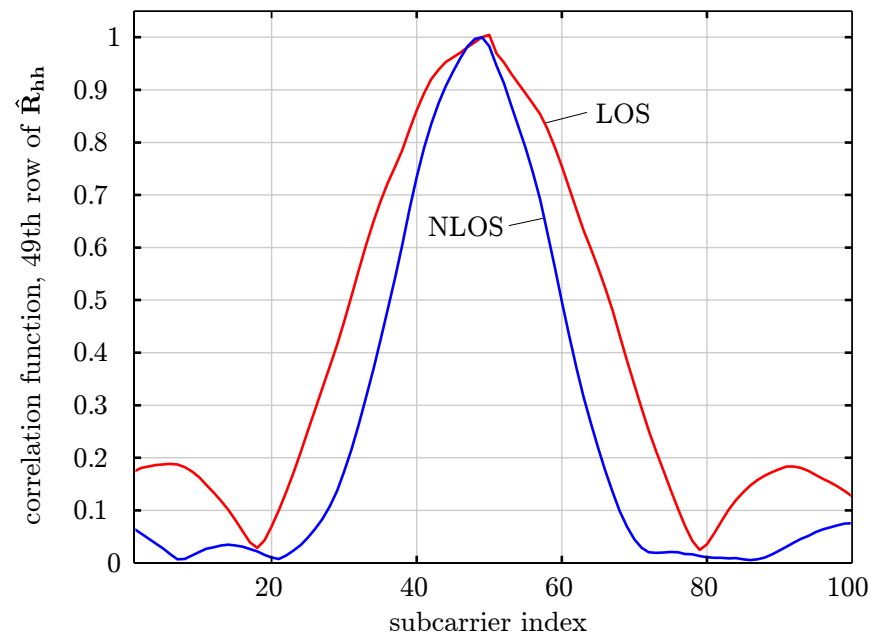

Fig. 1. Correlation between the 50-th subcarrier and all other subcarriers.

TABLE I

SNR IMPROVEMENT OVER THE LS CHANNEL ESTIMATOR.

\begin{tabular}{|l|c|c|}
\hline \multirow{2}{*}{ Channel estimator } & \multicolumn{2}{|c|}{ SNR gain [dB] at 10 Mbit/s } \\
\cline { 2 - 3 } & NLOS & LOS \\
\hline \hline perfect (genie driven) & $2.55 \mathrm{~dB}$ & $2.8 \mathrm{~dB}$ \\
LMMSE, $N_{c}=506$, Equ. (6) & $1.7 \mathrm{~dB}$ & $1.8 \mathrm{~dB}$ \\
ALMMSE, $L=10, N_{c}=506$, Equ. (12) & $1.6 \mathrm{~dB}$ & $1.7 \mathrm{~dB}$ \\
ALMMSE, $L=5, N_{c}=506$, Equ. (12) & $1.5 \mathrm{~dB}$ & $1.55 \mathrm{~dB}$ \\
ALMMSE, $L=5, N_{c}=1$, Equ. (12) & $1.4 \mathrm{~dB}$ & $1.55 \mathrm{~dB}$ \\
ALMMSE, $L=3, N_{c}=506$, Equ. (12) & $1.25 \mathrm{~dB}$ & $1.4 \mathrm{~dB}$ \\
ALMMSE, $L=2, N_{c}=506$, Equ. (12) & $0.1 \mathrm{~dB}$ & $1.05 \mathrm{~dB}$ \\
LS, Equ. (3) & $0 \mathrm{~dB}$ & $0 \mathrm{~dB}$ \\
\hline
\end{tabular}

channel did not change significantly within the transmission of the seven schemes [4]. For the throughput evaluation, we only count the number of bits in correctly received frames. We use an "optimum" AMC feedback mechanism, i.e. the AMC scheme that achieves the largest throughput for a specific channel realization at a specific transmit power level is selected. This is possible since all schemes were transmitted over a quasi static channel that did not change during the measurement at one receive antenna position.

\section{Vi. Measurement Results}

In this section, the results of our throughput measurements are presented.

All channel estimators were applied to the same receive data recorded in the previously described measurement campaign. Figure 1 shows the channel coefficient correlation between the 50-th subcarrier and all other subcarriers, i.e. the 50-th row of the correlation matrix $\hat{\mathbf{R}}_{\mathbf{h h}}$ (calculated according to Equation (7)). As expected, the LOS scenario (almost flat fading) shows slightly larger correlation than the NLOS scenario and thus larger coherence bandwidth. We can therefore also expect slightly better performance improvements of the LMMSE over the LS channel estimator.

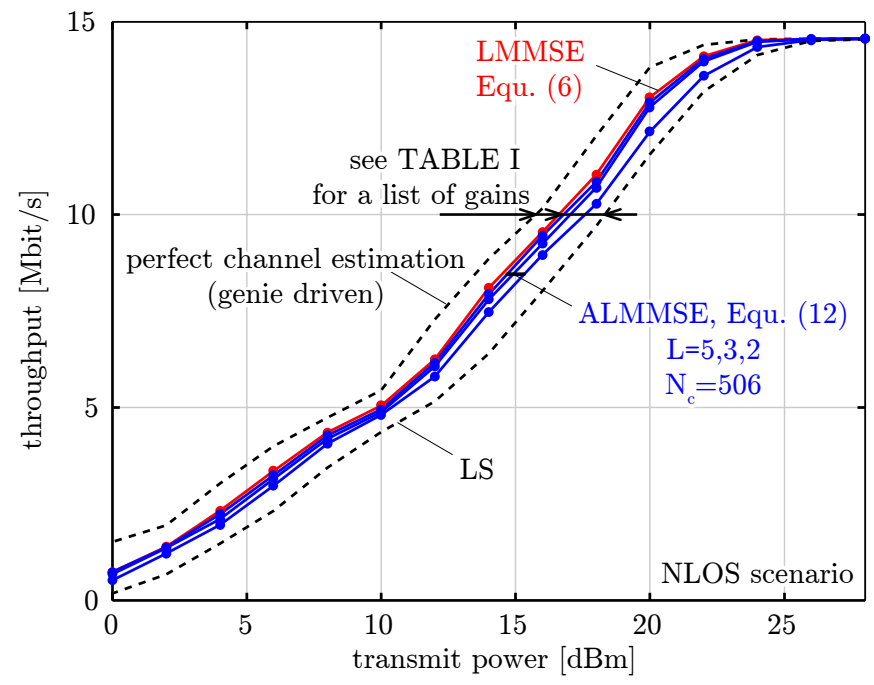

Fig. 2. Measured data throughput in the NLOS scenario for a $2 \times 2$ Alamouti space-time coded transmission system $\left(N_{c}=506\right)$.

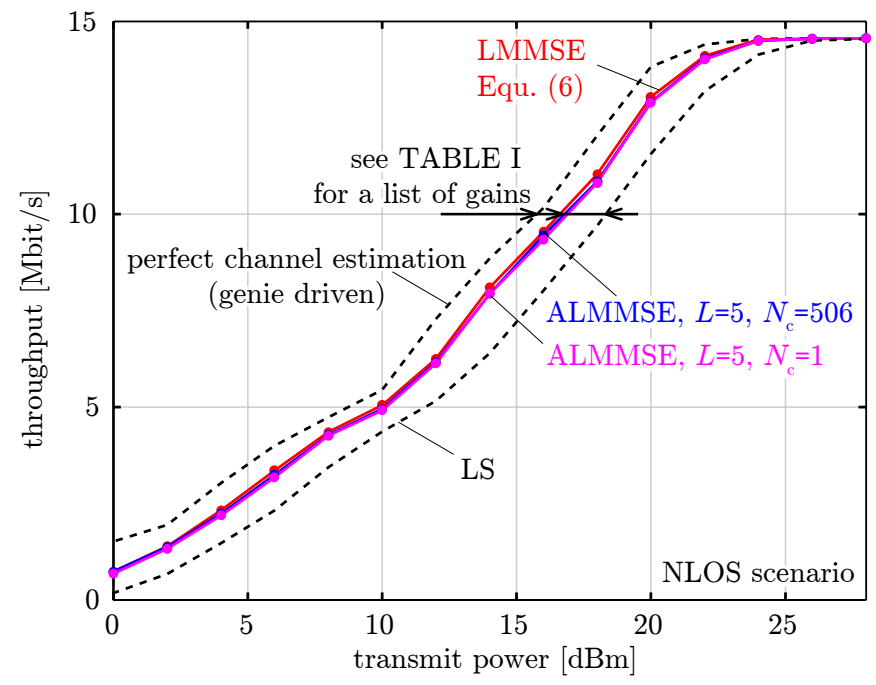

Fig. 3. Measured data throughput in the NLOS scenario for a $2 \times 2$ Alamouti space-time coded transmission system.

The measured data throughput for a $2 \times 2$ Alamouti spacetime coded transmission in the NLOS scenario is plotted in Figures 2 and 3. Figure 2 shows the results when the correlation matrices are obtained by averaging over all channel realizations in one scenario $\left(N_{c}=506\right)$. Figure 3 also includes the throughput, when the correlation matrix for the ALMMSE estimator is obtained from the single LS channel estimate $\left(N_{c}=1\right)$. The SNR gains of the channel estimators over the LS channel estimator are summarized in Table I for the NLOS and the LOS scenario. As expected, the performance of the ALMMSE channel estimator increases with the size of the channel correlation matrix. If a size of $L>5$ is selected the additional performance gain is only minor. We therefore conclude that $L=5$ provides a good performance/complexity tradeoff. Also, for $L=5$, our measurements results show that the use of a single channel realization $\left(N_{c}=1\right)$ in the corre- 
lation estimation costs only about $0.2 \mathrm{~dB}$ in SNR compared to an estimation that uses 506 channel realizations and also $L=5$. This small SNR loss for $L=5$ and $N_{c}=1$ makes a low complexity implementation of the ALMMSE channel estimator feasible and highly attractive.

\section{CONCLUSIONS}

In this paper we introduce a low complexity approximate LMMSE (ALMMSE) channel estimator. The ALMMSE estimator enhances the LS channel estimate by a spectral smoothing filter and achieves a performance close to the LMMSE estimator. The performance evaluation was carried out in terms of outdoor throughput measurements of a $2 \times 2$ Alamouti space-time coded WiMAX system. In such a transmission system, the ALMMSE estimator offers an SNR gain of about $1.5 \mathrm{~dB}$ over the LS estimator.

\section{ACKNOWLEDGMENT}

The authors would like to thank Michael Fischer, Lukas W. Mayer, and Arpad Scholtz for their help while preparing and conducting the measurements. We would also like to thank Martin Wrulich for his fruitful discussions and proofreading the paper.

This work has been funded by the Christian Doppler Laboratory for Design Methodology of Signal Processing Algorithms and the Austrian Research Centers GmbH.

\section{REFERENCES}

[1] IEEE, "IEEE standard for local and metropolitan area networks; part 16: Air interface for fixed broadband wireless access systems, IEEE Std. 802.16-2004," Oct. 2004. [Online]. Available: http://standards.ieee.org/getieee802/download/802.16-2004.pdf

[2] J.-J. van de Beek, O. Edfors, M. Sandell, S. K. Wilson, and P. O. Börjesson, "On channel estimation in OFDM systems," in Proc. IEEE Vehicular Technology Conference (VTC '95), vol. 2, Chicago, USA, 1995, pp. 815-819. [Online]. Available: http://ieeexplore.ieee.org/iel3/3758/10964/00504981. pdf?tp=\&arnumber $=504981$

[3] M. Noh, Y. Lee, and H. Park, "Low complexity LMMSE channel estimation for OFDM," IEE ProceedingsCommunications, vol. 153, no. 5, pp. 645-650, 2006. [Online]. Available: http://ieeexplore.ieee.org/iel5/2191/36095/01714642. pdf?tp=\&arnumber $=1714642$

[4] C. Mehlführer, S. Caban, and M. Rupp, "Experimental evaluation of adaptive modulation and coding in MIMO WiMAX with limited feedback," EURASIP Journal on Advances in Signal Processing, Special Issue on MIMO Systems with Limited Feedback, 2008 , in press.

[5] S. Caban, C. Mehlführer, R. Langwieser, A. L. Scholtz, and M. Rupp, "Vienna MIMO testbed," EURASIP Journal on Applied Signal Processing, vol. 2006, Article ID 54868, 2006. [Online]. Available: http://publik.tuwien.ac.at/files/pub-et_10929.pdf

[6] M. Rupp, C. Mehlführer, S. Caban, R. Langwieser, L. W. Mayer, and A. L. Scholtz, "Testbeds and rapid prototyping in wireless system design," EURASIP Newsletter, vol. 17, no. 3, pp. 32-50, Sept. 2006. [Online]. Available: http: //publik.tuwien.ac.at/files/pub-et_11232.pdf

[7] [Online]. Available: http://www.nt.tuwien.ac.at/wimaxsimulator/

[8] Kathrein, "Technical specification Kathrein antenna type no. 742 211." [Online]. Available: http://www.kathrein.de/de/mca/ produkte/download/9362108g.pdf 